\title{
ON THE BOUNDED INDEX PROPERTY FOR PRODUCTS OF ASPHERICAL POLYHEDRA
}

\author{
QIANG ZHANG AND SHENGKUI YE
}

\begin{abstract}
A compact polyhedron $X$ is said to have the Bounded Index Property for Homotopy Equivalences (BIPHE) if there is a finite bound $\mathcal{B}$ such that for any homotopy equivalence $f: X \rightarrow X$ and any fixed point class $\mathbf{F}$ of $f$, the index $|\operatorname{ind}(f, \mathbf{F})| \leq \mathcal{B}$. In this note, we consider the product of compact polyhedra, and give some sufficient conditions for it to have BIPHE. Moreover, we show that products of closed Riemannian manifolds with negative sectional curvature, in particular hyperbolic manifolds, have BIPHE, which gives an affirmative answer to a special case of a question asked by Boju Jiang.
\end{abstract}

\section{INTRODUCTION}

Fixed point theory studies fixed points of a self-map $f$ of a space $X$. Nielsen fixed point theory, in particular, is concerned with the properties of the fixed point set

$$
\operatorname{Fix} f:=\{x \in X \mid f(x)=x\}
$$

that are invariant under homotopy of the map $f$ (see [J1 for an introduction).

The fixed point set Fix $f$ splits into a disjoint union of fixed point classes: two fixed points $a$ and $a^{\prime}$ are in the same class if and only if there is a lifting $\tilde{f}: \widetilde{X} \rightarrow \widetilde{X}$ of $f$ such that $a, a^{\prime} \in p(\operatorname{Fix} \tilde{f})$, where $p: \widetilde{X} \rightarrow X$ is the universal cover. Let $\operatorname{Fpc}(f)$ denote the set of all the fixed point classes of $f$. For each fixed point class $\mathbf{F} \in \operatorname{Fpc}(f)$, a homotopy invariant index $\operatorname{ind}(f, \mathbf{F}) \in \mathbb{Z}$ is well-defined. A fixed point class is essential if its index is non-zero. The number of essential fixed point classes of $f$ is called the Nielsen number of $f$, denoted by $N(f)$. The famous Lefschetz-Hopf theorem says that the sum of the indices of the fixed points of $f$ is equal to the Lefschetz number $L(f)$, which is defined as

$$
L(f):=\sum_{q}(-1)^{q} \operatorname{Trace}\left(f_{*}: H_{q}(X ; \mathbb{Q}) \rightarrow H_{q}(X ; \mathbb{Q})\right) .
$$

In this note, all maps considered are continuous, and all spaces are triangulable, namely, they are homeomorphic to polyhedra. A compact polyhedron $X$ is said to have the Bounded Index Property (BIP)(resp. Bounded Index Property for Homeomorphisms (BIPH), Bounded Index Property for Homotopy Equivalences (BIPHE)) if there is an integer $\mathcal{B}>0$ such that for any map (resp. homeomorphism, homotopy equivalence) $f: X \rightarrow X$ and any fixed point class $\mathbf{F}$ of $f$, the index $|\operatorname{ind}(f, \mathbf{F})| \leq \mathcal{B}$. Clearly, if $X$ has BIP, then $X$ has BIPHE and hence has BIPH. For an aspherical

2010 Mathematics Subject Classification. 55M20, 55N10, $32 \mathrm{Q} 45$.

Key words and phrases. Bounded index property, fixed point, aspherical polyhedron, negative curved manifold, product.

The authors are partially supported by NSFC Grants \#11771345, \#11961131004 and \#11971389. 
closed manifold $M$, if the well-known Borel conjecture (any homotopy equivalence $f: M \rightarrow M$ is homotopic to a homeomorphism $g: M \rightarrow M)$ is true, then $M$ has BIPHE if and only if it has BIPH.

In [JG], Jiang and Guo proved that compact surfaces with negative Euler characteristics have BIPH. Later, Jiang [J2] showed that graphs and surfaces with negative Euler characteristics not only have BIPH but also have BIP (see [K1, K2] and [JWZ for some parallel results). Moreover, Jiang asked the following question:

Question 1.1. ([J2, Qusetion 3]) Does every compact aspherical polyhedron $X$ (i.e. $\pi_{i}(X)=0$ for all $i>1$ ) have BIP or BIPH?

In $[\mathrm{Mc}$, McCord showed that infrasolvmanifolds (manifolds which admit a finite cover by a compact solvmanifold) have BIP. In [JW], Jiang and Wang showed that geometric 3-manifolds have BIPH for orientation-preserving self-homeomorphisms: the index of each essential fixed point class is \pm 1 . In [Z1, the first author showed that orientable compact Seifert 3-manifolds with hyperbolic orbifolds have BIPH, and later in [Z2, Z3], he showed that compact hyperbolic $n$-manifolds (not necessarily orientable) also have BIPH. Recently, in [ZZ, Zhang and Zhao showed that products of hyperbolic surfaces have BIPH.

Note that in [J2, Section 6], Jiang gave an example that showed that BIPH is not preserved by taking products: the 3 -sphere $S^{3}$ has BIPH while the product $S^{3} \times S^{3}$ does not have BIPH. In this note, we consider the product of connected compact polyhedra, and give some sufficient conditions for it to have BIPHE (and hence has BIPH). The main result of this note is the following:

Theorem 1.2. Suppose $X_{1}, \ldots, X_{n}$ are connected compact aspherical polyhedra satisfying the following two conditions:

(1) $\pi_{1}\left(X_{i}\right) \neq \pi_{1}\left(X_{j}\right)$ for $i \neq j$, and all of them are centerless and indecomposable;

(2) all of $X_{1}, \ldots, X_{n}$ have BIPHE.

Then the product $X_{1} \times \cdots \times X_{n}$ also has BIPHE (and hence has BIPH).

Moreover, we show that products of closed Riemannian manifolds with negative sectional curvature have BIPHE:

Theorem 1.3. Let $M=M_{1} \times \cdots \times M_{n}$ be a product of finitely many connected closed Riemannian manifolds, each with negative sectional curvature everywhere but not necessarily with the same dimensions (in particular hyperbolic manifolds). Then $M$ has BIPHE.

Recall that a closed 2-dimensional Riemannian manifold $M$ with negative sectional curvature is a closed hyperbolic surface, and hyperbolic surfaces have BIP. As a corollary of Theorem 1.3, we have

Corollary 1.4. A closed Riemannian manifold with negative sectional curvature everywhere has BIPHE.

To prove the above theorems, we first study automorphisms of products of groups in Section 2, and give some facts about the bounded index property of fixed points in Section 3. Then in Section 4, we generalize the results of alternating homeomorphisms (see [ZZ, Section 3]) to that of cyclic homeomorphisms of products of surfaces. Finally in Section 5 , we show that every homotopy equivalence of products of aspherical manifolds can be homotoped to two nice forms, and taking advantage of that, we finish the proofs. 


\section{Automorphisms of Products of Groups}

In this section, we give some facts about automorphisms of direct products of finitely many groups.

Definition 2.1. A group $G$ is called unfactorizable if whenever $G=H K$ for subgroups $H, K$ satisfying $h k=k h$ for any $h \in H, k \in K$ we have either $H=1$ or $K=1$. If $G=H \times K$ for some groups $H, K$ implies either $H$ or $K$ is trivial, we call $G$ is indecomposable.

Lemma 2.2. Let $G$ be an unfactorizable group and $G=\prod_{i=1}^{n} A_{i}$ a direct product. Then $A_{i}=G$ for some $i$ and all $A_{j}=1$ for $i \neq j$.

Proof. Suppose that there are at least two non-trivial components $A_{1}, A_{2}$. Then $G=A_{1} A_{2} \times \Pi_{i \neq 1,2} A_{i}$. Then $\Pi_{i \neq 1,2} A_{i}=1$, since $G$ is unfactorizable. Therefore, $G=A_{1} A_{2}$ a contradiction.

Lemma 2.3. A group $G$ is unfactorizable if and only if $G$ is centerless and indecomposable.

Proof. Suppose that $G$ is unfactorizable. Since $G=G C$ for the center subgroup $C$, we see that $C=1$. An unfactorizable group is obviously indecomposable. Conversely, assume that $G$ is centerless and indecomposable. If $G=K H$ for commuting $K, H$. Then any element $g \in K \cap H$ is central and thus trivial. This implies that $G=K \times H$. Therefore, either $K$ or $H$ is trivial.

For a direct product $G=G_{1} \times \cdots \times G_{n}$, we collect together the coordinates corresponding to isomorphic $G_{i}$ 's and present it in the form $G=G_{1}^{n_{1}} \times \cdots \times G_{m}^{n_{m}}$, where $n_{i} \geq 1$ and $G_{i} \not G_{j}$ for $1 \leq i \neq j \leq m$. Given automorphisms $\phi_{i} \in$ $\operatorname{Aut}\left(G_{i}\right)$ for $i=1, \ldots, n$, let $\prod_{i=1}^{n} \phi_{i}=\phi_{1} \times \cdots \times \phi_{n}: G \rightarrow G,\left(g_{1}, \ldots, g_{n}\right) \mapsto$ $\left(\phi_{1}\left(g_{1}\right), \ldots, \phi_{n}\left(g_{n}\right)\right)$ be the product of $\phi_{i}$ 's. We have an analogous result of [ZVW] Proposition 4.4] as follows.

Proposition 2.4. Let each group $G_{i}, i=1, \ldots, m$, be unfactorizable, and $G=$ $G_{1}^{n_{1}} \times \cdots \times G_{m}^{n_{m}}$ a direct product, where $m \geq 1, n_{i} \geq 1$, and $G_{i} \not G_{j}$ for $i \neq j$. Then for every $\phi \in \operatorname{Aut}(G)$, there exist automorphisms $\phi_{i, j} \in \operatorname{Aut}\left(G_{i}\right)$ and permutations $\sigma_{i} \in S_{n_{i}}$, such that

$$
\phi=\sigma_{1} \circ \cdots \circ \sigma_{m} \circ\left(\prod_{i=1}^{m} \prod_{j=1}^{n_{i}} \phi_{i, j}\right)=\prod_{i=1}^{m}\left(\sigma_{i} \circ \prod_{j=1}^{n_{i}} \phi_{i, j}\right) .
$$

Proof. For brevity, we first assume that $G=G_{1} \times G_{2}$ (here $G_{1}$ may be isomorphic to $G_{2}$ ), and $\phi: G_{1} \times G_{2} \rightarrow G_{1} \times G_{2}$ an automorphism. Let

$$
A_{1}=\left\{a_{1} \mid \phi\left(g_{1}, 1\right)=\left(a_{1}, b_{1}\right), g_{1} \in G_{1}\right\}, B_{1}=\left\{b_{1} \mid \phi\left(g_{1}, 1\right)=\left(a_{1}, b_{1}\right), g_{1} \in G_{1}\right\},
$$

and

$$
A_{2}=\left\{a_{2} \mid \phi\left(1, g_{2}\right)=\left(a_{2}, b_{2}\right), g_{2} \in G_{2}\right\}, B_{2}=\left\{b_{2} \mid \phi\left(1, g_{2}\right)=\left(a_{2}, b_{2}\right), g_{2} \in G_{2}\right\} .
$$

Then $G_{1}=A_{1} A_{2}, a_{1} a_{2}=a_{2} a_{1}$ for every $a_{i} \in A_{i}$; and $G_{2}=B_{1} B_{2}, b_{1} b_{2}=b_{2} b_{1}$ for every $b_{i} \in B_{i}$. Since $G_{1}, G_{2}$ are unfactorizable, we see that either $A_{1}$ or $A_{2}$ is trivial, and either $B_{1}$ or $B_{2}$ is trivial.

If $A_{1}=G_{1}, A_{2}=1$, then $B_{1}=1, B_{2}=G_{2}$, and we have

$$
\phi=\phi_{1} \times \phi_{2}: G_{1} \times G_{2} \rightarrow G_{1} \times G_{2}, \quad\left(g_{1}, g_{2}\right) \mapsto\left(\phi_{1}\left(g_{1}\right), \phi_{2}\left(g_{2}\right)\right)=\left(a_{1}, b_{2}\right)
$$


where $\phi_{i} \in \operatorname{Aut}\left(G_{i}\right)$.

If $A_{1}=1, A_{2}=G_{1}$, then $B_{1}=G_{2}, B_{2}=1$, then we have $G_{1} \cong G_{2}$, and

$\phi=\sigma \circ\left(\phi_{1} \times \phi_{2}\right): G_{1} \times G_{2} \rightarrow G_{1} \times G_{2}, \quad\left(g_{1}, g_{2}\right) \mapsto\left(\phi_{2}\left(g_{2}\right), \phi_{1}\left(g_{1}\right)\right)=\left(a_{2}, b_{1}\right)$

for $\phi_{i} \in \operatorname{Aut}\left(G_{i}\right)$ and $\sigma \in S_{2}$ a permutation.

Now we have proved that Proposition 2.4 holds for the case $G=G_{1} \times G_{2}$. For the general case that $G=\prod_{i} G_{i}$ has more than 2 factors, the same argument as above shows that Proposition 2.4 also holds.

Since the inner automorphism group $\operatorname{Inn}\left(\prod_{i} G_{i}\right)$ of a product is isomorphic to the product $\prod_{i} \operatorname{Inn}\left(G_{i}\right)$, summarizing the above results, we have proved the following:

Theorem 2.5. Let $G_{1}, \ldots, G_{m}$ be finitely many centerless indecomposable groups, and $G_{i} \supsetneqq G_{j}$ for $i \neq j$. Then the automorphism group of the product $\prod_{i=1}^{m} G_{i}^{n_{i}}$

$$
\operatorname{Aut}\left(\prod_{i=1}^{m} G_{i}^{n_{i}}\right) \cong \prod_{i=1}^{m}\left(\prod_{n_{i}} \operatorname{Aut}\left(G_{i}\right)\right) \rtimes S_{n_{i}}
$$

and the outer automorphism group

$$
\operatorname{Out}\left(\prod_{i=1}^{m} G_{i}^{n_{i}}\right) \cong \prod_{i=1}^{m}\left(\prod_{n_{i}} \operatorname{Out}\left(G_{i}\right)\right) \rtimes S_{n_{i}}
$$

where the symmetric group $S_{n_{i}}$ of $n_{i} \geq 1$ elements acts on $\prod_{n_{i}} \operatorname{Aut}\left(G_{i}\right)$ and $\prod_{n_{i}} \operatorname{Out}\left(G_{i}\right)$ by natural permutations.

There are many examples of centerless indecomposable groups.

Example 2.6. Non-abelian free groups are centerless indecomposable groups.

Example 2.7. Non-abelian torsion-free Gromov hyperbolic groups are centerless indecomposable groups. In particular, the fundamental group of a closed Riemannian manifold with negative sectional curvature everywhere is centerless and indecomposable.

Proof. Let $G$ be a non-abelian torsion-free Gromov hyperbolic group and $1 \neq \gamma \in$ $G$. It is well-known that the centralizer subgroup $C(\gamma)=\{g \in G \mid g \gamma=\gamma g\}$ contains $\langle\gamma\rangle$ as a finite-index subgroup (see $[\mathrm{BH}]$, Corollary 3.10, p.462). Therefore, the center of $G$ is trivial and $G$ is indecomposable.

\section{Facts about the Bounded index PRoperties}

In this section, we give some facts about BIP, BIPHE and BIPH. In order to state results conveniently, we will use the following definition.

Definition 3.1. Let $X$ be a compact polyhedron and $C$ be a family of self-maps of $X$. We call that $X$ has the Bounded Index Property with respect to $C$ (denoted by BIPC) if there exists an integer $\mathcal{B}>0$ (depending only on $C$ ) such that for any map $f \in C$ and any fixed point class $\mathbf{F}$ of $f$, the index $|\operatorname{ind}(f, \mathbf{F})| \leq \mathcal{B}$. The minimum such a $\mathcal{B}$ is called the bounded index for $C$.

When $C$ is the set of all self-maps (self-homeomorphisms, self-homotopy equivalences, respectively), we simply denote the BIPC by BIP (BIPH, BIPHE, respectively). It is obvious that $\mathrm{BIPC}_{1}$ implies $\mathrm{BIPC}_{2}$ when $C_{2}$ is a subset of $C_{1}$.

For maps of polyhedra, Jiang gave the following definition of mutant, and showed that the Nielsen fixed point invariants are invariants of mutants (see [J2, Sect. 1]). 
Definition 3.2. Let $f: X \rightarrow X$ and $g: Y \rightarrow Y$ be self-maps of compact connected polyhedra. We say $g$ is obtained from $f$ by commutation, if there exist maps $\phi: X \rightarrow Y$ and $\psi: Y \rightarrow X$ such that $f=\psi \circ \phi$ and $g=\phi \circ \psi$. Say $g$ is a mutant of $f$, if there is a finite sequence $\left\{f_{i}: X_{i} \rightarrow X_{i} \mid i=1,2, \cdots, k\right\}$ of self-maps of compact polyhedra such that $f=f_{1}, g=f_{k}$, and for each $i$, either $X_{i+1}=X_{i}$ and $f_{i+1} \simeq f_{i}$, or $f_{i+1}$ is obtained from $f_{i}$ by commutation.

Lemma 3.3 (Jiang, J22). Mutants have the same set of indices of essential fixed point classes, hence also the same Lefschetz number and Nielsen number.

Note that mutants give an equivalence relation on self-maps of compact polyhedra. In other words, two self-maps $f: X \rightarrow X$ and $g: Y \rightarrow Y$ are mutant-equivalent if there exist finitely many maps of compact polyhedra $X_{1}=X, X_{2}, \ldots, X_{k}=Y$,

$$
u_{i}: X_{i} \rightarrow X_{i+1}, \quad v_{i}: X_{i+1} \rightarrow X_{i}, \quad i=1,2, \cdots, k-1,
$$

such that $f \simeq v_{1} \circ u_{1}: X_{1} \rightarrow X_{1}, g \simeq u_{k-1} \circ v_{k-1}: X_{k} \rightarrow X_{k}$, and for $i<k-1$,

$$
u_{i} \circ v_{i} \simeq v_{i+1} \circ u_{i+1} \text {. }
$$

For a self-map $f: X \rightarrow X$ of a connected compact polyhedron $X$, let $[f]_{m}$ denote the mutant-equivalent class of $f$, and for a family $C$ of self-maps of $X$, let $[C]_{m}:=\left\{[f]_{m} \mid f \in C\right\}$ be the set of mutant-equivalent classes of $C$. Note that $f$ has only finitely many non-empty fixed point classes, and each is a compact subset of $X$, we have a finite bound $\mathcal{B}_{f}$ of ind $(f, \mathbf{F})$ for all the fixed point classes $\mathbf{F}$ of $f$. As an immediate consequence of Lemma 3.3 , we have the following:

Proposition 3.4. For any family $C$ of self-maps of a connected compact polyhedron $X$, the set $\{\operatorname{ind}(f, \mathbf{F}) \mid f \in C, \mathbf{F} \in \operatorname{Fpc}(f)\}$ defined on $C$ factors through the equivalence classes $[C]_{m}$. Namely, for any set $[C]_{m}$ of mutant-equivalent classes, we have a set of indices

$$
\operatorname{ind}\left([C]_{m}\right):=\{\operatorname{ind}(f, \mathbf{F}) \mid f \in C, \mathbf{F} \in \operatorname{Fpc}(f)\}
$$

depending only on $[C]_{m}$. Moreover, if $[C]_{m}$ is finite, then $X$ has BIPC.

Suppose that $X$ is aspherical and let $[X]$ (resp. $[X]_{h . e}$ ) be the set of all homotopy classes of self-maps (resp. self-homotopy equivalences) of $X$. It is well-known that there is a bijective correspondence

$$
\eta:[X] \longleftrightarrow \operatorname{End}\left(\pi_{1}(X)\right) / \operatorname{Inn}\left(\pi_{1}(X)\right),
$$

given by sending a self-map $f$ to the induced endomorphism $f_{\pi}$ of the fundamental group, where the inner automorphism group $\operatorname{Inn}\left(\pi_{1}(X)\right)$ acts on the semigroup $\operatorname{End}\left(\pi_{1}(X)\right)$ of all the endomorphisms of $\pi_{1}(X)$ by composition. Note that $\eta$ induces a bijection (still denoted by $\eta$ )

$$
\eta:[X]_{h . e} \longleftrightarrow \operatorname{Out}\left(\pi_{1}(X)\right)
$$

For any self-map $f$ of $X$, let $[f]$ denote the homotopy class of $f$, and for any family $C$ of self-homotopy equivalences of $X$, let $[C]:=\{[f] \mid f \in C\}$ be the set of homotopy classes of $C$. Then the image $\eta([C])$ is a subset of $\operatorname{Out}\left(\pi_{1}(X)\right)$. For a group $G$ and a subset $H$ of $G$, two elements $g, h \in H$ are conjugate if there exists an element $k \in G$ such that $g=k h k^{-1}$. Let $\bar{h}$ be the conjugacy class of $h$ in $G$, and $\operatorname{Conj}_{G} H:=\{\bar{h} \mid h \in H\}$ be the set of conjugacy classes of $H$ in $G$. We have the following: 
Lemma 3.5. Let $X$ be a connected compact aspherical polyhedron. Suppose that $C$ is a family of self-homotopy equivalences of $X$. Then we have a natural surjection

$$
\Phi: \operatorname{Conj}_{\operatorname{Out}\left(\pi_{1}(X)\right)} \eta([C]) \longrightarrow[C]_{m}, \quad \text { defined by } \overline{\eta([f])} \longmapsto[f]_{m} .
$$

Moreover, if the set $\operatorname{Conj}_{\mathrm{Out}\left(\pi_{1}(X)\right)} \eta([C])$ is finite, then $[C]_{m}$ is also finite and hence $X$ has BIPC. In particular, when $\operatorname{Out}\left(\pi_{1}(X)\right)$ has finitely many conjugacy classes, the polyhedron $X$ has the bounded index property with respect to the set of all selfhomotopy equivalences, i.e., $X$ has BIPHE.

Proof. For self-homotopy equivalences $f, g \in C$, if $\overline{\eta([f])}=\overline{\eta([g])}$, then there exists $\eta([h])=\eta\left(\left[h^{\prime}\right]\right)^{-1} \in \operatorname{Out}\left(\pi_{1}(X)\right)$ for $h: X \rightarrow X$ a homotopy equivalence and $h^{\prime}: X \rightarrow X$ a homotopy inverse of $h$ such that

$$
\eta([g])=\eta([h]) \cdot \eta([f]) \cdot \eta([h])^{-1}=\eta([h]) \cdot \eta([f]) \cdot \eta\left(\left[h^{\prime}\right]\right) \in \operatorname{Out}\left(\pi_{1}(X)\right) .
$$

This implies that $g \simeq h \circ\left(f \circ h^{\prime}\right)$. Note that $f \simeq\left(f \circ h^{\prime}\right) \circ h$, so we have $[f]_{m}=[g]_{m}$. Therefore, $\Phi$ is well-defined. It is obvious from the definition that $\Phi$ is surjective, and the proof is finished by Proposition 3.4

Theorem 3.6. Let $M=M_{1} \times \cdots \times M_{m}$ be a product of finitely many connected closed Riemannian manifolds, each with negative sectional curvature everywhere, and with (not necessarily the same) dimension $\geq 3$. Then $M$ has BIPHE.

Proof. Rips and Sela RS building on ideas of Paulin proved that $\operatorname{Out}(G)$ is a finite group when $G$ is the fundamental group of a closed Riemannian manifolds of dimension $\geq 3$ with negative sectional curvature everywhere. Since the factors $M_{i}^{n_{i}}, i=1, \ldots, m$, are closed Riemannian manifolds, each with negative sectional curvature everywhere, and the dimensions $n_{i} \geq 3$, we have that $\operatorname{Out}\left(\pi_{1}(M)\right)$ is also finite by Theorem 2.5. Therefore, $M$ has BIPHE (and hence BIPH) by Lemma 3.5 .

\section{Fixed points of CyCliC homeomorphisms of products of SuRfaces}

In this section, we will generalize the results of alternating homeomorphisms (see [ZZ, Section 3]) to cyclic homeomorphisms of products of surfaces. Let $F$ be a connected closed hyperbolic surface, and hence, the Euler characteristics $\chi(F)<0$.

Definition 4.1. A self-homeomorphism $f$ of $F^{m}:=\overbrace{F \times \cdots \times F}^{m}$, is called a cyclic homeomorphism, if

$f=\tau \circ\left(\prod_{i=1}^{m} f_{i}\right): F^{m} \rightarrow F^{m},\left(a_{1}, a_{2}, \ldots, a_{m}\right) \mapsto\left(f_{m}\left(a_{m}\right), f_{1}\left(a_{1}\right), \ldots, f_{m-1}\left(a_{m-1}\right)\right)$,

where $f_{1}, \ldots, f_{m}$ are self-homeomorphisms of $F$, and $\tau=(12 \cdots m) \in S_{m}$ is a $m$-cycle.

Note that for a compact hyperbolic surface, every homeomorphism is isotopic to a diffeomorphism, then by the same argument as in the proof of [ZZ, Lemma 3.2], we have

Lemma 4.2. Let $f_{1}, \ldots, f_{m}$ be self-homeomorphisms of $F$, and $f=\tau \circ\left(\prod_{i=1}^{m} f_{i}\right)$ : $F^{m} \rightarrow F^{m}$ a cyclic homeomorphism. Then $f_{1}, \ldots, f_{m}$ can be isotoped to diffeomorphisms $g_{1}, \ldots, g_{m}$ respectively, such that the graph of the corresponding cyclic homeomorphism $g=\tau \circ\left(\prod_{i=1}^{m} g_{i}\right): F^{m} \rightarrow F^{m}$ is transversal to the diagonal in 
$F^{m}$. Moreover, $f$ is homotopic to $g$ and, for each fixed point $\left(a_{1}, a_{2}, \ldots, a_{m}\right)$ of $g$, there are charts of $F^{m}$ at $\left(a_{1}, a_{2}, \ldots, a_{m}\right)$ such that under the charts, $g$ has a local canonical form

$$
\begin{array}{ll} 
& \left(u_{11}, u_{12}, u_{21}, u_{22}, \ldots, u_{m 1}, u_{m 2}\right) \\
\mapsto \quad & \left(g_{m 1}\left(u_{m 1}, u_{m 2}\right), g_{m 2}\left(u_{m 1}, u_{m 2}\right), g_{11}\left(u_{11}, u_{12}\right), g_{12}\left(u_{11}, u_{12}\right),\right. \\
& \left.\ldots, g_{(m-1) 1}\left(u_{(m-1) 1}, u_{(m-1) 2}\right), g_{(m-1) 2}\left(u_{(m-1) 1}, u_{(m-1) 2}\right)\right)
\end{array}
$$

where $g_{i 1}, g_{i 2}$ are the components of $g_{i}$ under the charts.

Lemma 4.3. If $f=\tau \circ\left(\prod_{i=1}^{m} f_{i}\right): F^{m} \rightarrow F^{m}$ is a cyclic homeomorphism, then the natural map

$$
\rho: F \rightarrow F^{m}, \quad a_{1} \mapsto\left(a_{1}, f_{1}\left(a_{1}\right), \ldots, f_{m-1} \circ \cdots \circ f_{2} \circ f_{1}\left(a_{1}\right)\right)
$$

induces an index-preserving one-to-one corresponding between the set $\operatorname{Fpc}\left(f_{m} \circ \cdots \circ\right.$ $\left.f_{2} \circ f_{1}\right)$ of fixed point classes of $f_{m} \circ \cdots \circ f_{2} \circ f_{1}$ and the set $\operatorname{Fpc}(f)$ of fixed point classes of $f$.

Proof. It is clear that

$$
\begin{aligned}
\operatorname{Fix} f & =\left\{\left(a_{1}, a_{2}, \ldots, a_{m}\right) \mid f_{1}\left(a_{1}\right)=a_{2}, f_{2}\left(a_{2}\right)=a_{3}, \ldots, f_{m}\left(a_{m}\right)=a_{1}\right\} \\
& =\left\{\left(a_{1}, f_{1}\left(a_{1}\right), \ldots, f_{m-1} \circ \cdots \circ f_{2} \circ f_{1}\left(a_{1}\right)\right) \mid a_{1} \in \operatorname{Fix}\left(f_{m} \circ \cdots \circ f_{2} \circ f_{1}\right)\right\} .
\end{aligned}
$$

Suppose that $a_{1}$ and $a_{1}^{\prime}$ are in the same fixed point class of $f_{m} \circ \cdots \circ f_{1}$, and $p: \widetilde{F} \rightarrow F$ is the universal cover. Then there is a lifting $\tilde{f}_{i}$ of $f_{i}$ such that $a_{1}, a_{1}^{\prime} \in$ $p\left(\operatorname{Fix}\left(\tilde{f}_{m} \circ \cdots \circ \tilde{f}_{1}\right)\right)$, and there is a point $\tilde{a}_{1} \in p^{-1}\left(a_{1}\right)$ and a point $\tilde{a}_{1}^{\prime-1}\left(a_{1}^{\prime}\right)$ with $\left(\tilde{f}_{m} \circ \cdots \circ \tilde{f}_{1}\right)\left(\tilde{a}_{1}\right)=\tilde{a}_{1}$ and $\left(\tilde{f}_{m} \circ \cdots \circ \tilde{f}_{1}\right)\left(\tilde{a}_{1}^{\prime}\right)=\tilde{a}_{1}^{\prime}$. Hence,

$$
\begin{aligned}
& \left(\tau \circ\left(\tilde{f}_{1} \times \cdots \times \tilde{f}_{m}\right)\right)\left(\tilde{a}_{1}, \tilde{f}_{1}\left(\tilde{a}_{1}\right), \ldots, \tilde{f}_{m-1} \circ \cdots \circ \tilde{f}_{1}\left(\tilde{a}_{1}\right)\right) \\
= & \left.\left(\left(\tilde{f}_{m} \circ \cdots \circ \tilde{f}_{1}\right)\left(\tilde{a}_{1}\right), \tilde{f}_{1}\left(a_{1}\right), \ldots, \tilde{f}_{m-1} \circ \cdots \circ \tilde{f}_{1}\right)\left(\tilde{a}_{1}\right)\right) \\
= & \left(\tilde{a}_{1}, \tilde{f}_{1}\left(\tilde{a}_{1}\right), \ldots, \tilde{f}_{m-1} \circ \cdots \circ \tilde{f}_{1}\left(\tilde{a}_{1}\right)\right) .
\end{aligned}
$$

It follows that

$$
\left(a_{1}, f_{1}\left(a_{1}\right), \ldots, f_{m-1} \circ \cdots \circ f_{1}\left(a_{1}\right)\right) \in\left(\prod_{m} p\right)\left(\operatorname{Fix}\left(\tau \circ\left(\tilde{f}_{1} \times \cdots \times \tilde{f}_{m}\right)\right)\right) .
$$

Similarly, we also have

$$
\left(a_{1}^{\prime}, f_{1}\left(a_{1}^{\prime}\right), \ldots, f_{m-1} \circ \cdots \circ f_{1}\left(a_{1}^{\prime}\right)\right) \in\left(\prod_{m} p\right)\left(\operatorname{Fix}\left(\tau \circ\left(\tilde{f}_{1} \times \cdots \times \tilde{f}_{m}\right)\right)\right) .
$$

Since $\left(\tau \circ\left(\tilde{f}_{1} \times \cdots \times \tilde{f}_{m}\right)\right)$ is a lifting of $f$, we obtain that $\left(a_{1}, f_{1}\left(a_{1}\right), \ldots, f_{m-1} \circ\right.$ $\left.\cdots \circ f_{1}\left(a_{1}\right)\right)$ and $\left(a_{1}^{\prime}, f_{1}\left(a_{1}^{\prime}\right), \ldots, f_{m-1} \circ \cdots \circ f_{1}\left(a_{1}^{\prime}\right)\right)$ are in the same fixed point class of $f$. Conversely, suppose that the two points above are in the same fixed point class of $f$. Then there is a lifting $\tilde{f}_{i}$ of $f_{i}$ such that both of them lie in $\left(\prod_{m} p\right)\left(\operatorname{Fix}\left(\tau \circ\left(\tilde{f}_{1} \times \cdots \times \tilde{f}_{m}\right)\right)\right)$. Hence, $a_{1}, a_{1}^{\prime} \in p\left(\operatorname{Fix}\left(\tilde{f}_{m} \circ \cdots \circ \tilde{f}_{1}\right)\right)$, we conclude that $a_{1}$ and $a_{1}^{\prime}$ are in the same fixed point class of $f_{m} \circ \cdots \circ f_{2} \circ f_{1}$.

Now we shall prove that as a bijective correspondence between the sets of fixed point classes, $\rho$ is index-preserving. Since the indices of fixed point classes are invariant under homotopies, by Lemma 4.2 we may homotope $f_{i}$ for $i=1,2, \ldots, m$ such that the graph of $f$ is transversal to the diagonal, and $f$ has local canonical forms in a neighborhood of every fixed point. Suppose that the differential $D f_{i}$ of 
$f_{i}$ at $a_{i}$ is $N_{i}=\left(\begin{array}{ll}\frac{\partial f_{i 1}}{\partial u_{i 1}} & \frac{\partial f_{i 1}}{\partial u_{i 2}} \\ \frac{\partial f_{i 2}}{\partial u_{i 1}} & \frac{\partial f_{i 2}}{\partial u_{i 2}}\end{array}\right)$. Then the differential $D f$ of $f$ at $\left(a_{1}, a_{2}, \ldots, a_{m}\right)$ is

$$
N=\left(\begin{array}{ccccc}
0 & 0 & \cdots & 0 & N_{m} \\
N_{1} & 0 & \cdots & 0 & 0 \\
0 & N_{2} & \cdots & 0 & 0 \\
\vdots & \vdots & \ddots & \vdots & \vdots \\
0 & 0 & \cdots & N_{m-1} & 0
\end{array}\right)
$$

Therefore, the index of $f_{m} \circ \cdots \circ f_{2} \circ f_{1}$ at the fixed point $a_{1}$ is

$$
\operatorname{ind}\left(f_{m} \circ \cdots \circ f_{2} \circ f_{1}, a_{1}\right)=\operatorname{sgn} \operatorname{det}\left(I_{2}-N_{m} \cdots N_{2} N_{1}\right),
$$

and the index of $f$ at the fixed point $\left(a_{1}, a_{2}, \ldots, a_{m}\right)$ is

$$
\begin{aligned}
\operatorname{ind}\left(f,\left(a_{1}, a_{2}, \ldots, a_{m}\right)\right) & =\operatorname{sgn} \operatorname{det}\left(I_{2 m}-N\right) \\
& =\operatorname{sgn} \operatorname{det}\left(I_{2}-N_{m-1} \cdots N_{1} N_{m}\right) \\
& =\operatorname{sgn} \operatorname{det}\left(I_{2}-N_{m} \cdots N_{2} N_{1}\right),
\end{aligned}
$$

where $I_{k}$ is the identity matrix of order $k$. Therefore,

$$
\operatorname{ind}\left(f_{m} \circ \cdots \circ f_{2} \circ f_{1}, a_{1}\right)=\operatorname{ind}\left(f,\left(a_{1}, a_{2}, \ldots, a_{m}\right)\right),
$$

and the proof is finished.

As a corollary, we have

\section{Corollary 4.4 .}

$$
N(f)=N\left(f_{m} \circ \cdots \circ f_{2} \circ f_{1}\right), \quad L(f)=L\left(f_{m} \circ \cdots \circ f_{2} \circ f_{1}\right) .
$$
have

Directly following from Lemma 4.3, Corollary 4.4 and [JG, Theorem 4.1], we

Proposition 4.5. If $f: F^{m} \rightarrow F^{m}$ is a cyclic homeomorphism, then

(A) For every fixed point class $\mathbf{F}$ of $f$, we have

$$
2 \chi(F)-1 \leq \operatorname{ind}(f, \mathbf{F}) \leq 1
$$

Moreover, almost every fixed point class $\mathbf{F}$ of $f$ has index $\geq-1$, in the sense that

$$
\sum_{\operatorname{ind}(f, \mathbf{F})<-1}\{\operatorname{ind}(f, \mathbf{F})+1\} \geq 2 \chi(F)
$$

where the sum is taken over all fixed point classes $\mathbf{F}$ with $\operatorname{ind}(f, \mathbf{F})<-1$;

(B) Let $L(f)$ and $N(f)$ be the Lefschetz number and the Nielsen number of $f$ respectively. Then

$$
|L(f)-\chi(F)| \leq N(f)-\chi(F)
$$

\section{Fixed points of product maps and proofs of Theorem 1.2 and 1.3}

To prove Theorem 1.2 and Theorem 1.3 , we need some facts about fixed points of product maps. 
5.1. Fixed points of product maps. Let $X_{1}, \ldots, X_{n}$ be connected compact polyhedra.

Definition 5.1. A self-map $f: X_{1} \times \cdots \times X_{n} \rightarrow X_{1} \times \cdots \times X_{n}$ is called a product map, if

$f=f_{1} \times \cdots \times f_{n}: X_{1} \times \cdots \times X_{n} \rightarrow X_{1} \times \cdots \times X_{n}, \quad\left(a_{1}, \ldots, a_{n}\right) \mapsto\left(f_{1}\left(a_{1}\right), \ldots, f_{n}\left(a_{n}\right)\right)$, where $f_{i}$ is a self-map of $X_{i}, i=1, \ldots, n$.

By a proof analogous to that of [ZZ, Lemma 2.2], we have the following lemma about the fixed point classes of product maps.

Lemma 5.2. If $f: X_{1} \times \cdots \times X_{n} \rightarrow X_{1} \times \cdots \times X_{n}$ is a product map, then $\operatorname{Fix} f=\operatorname{Fix} f_{1} \times \cdots \times \operatorname{Fix} f_{n}$, and each fixed point class $\mathbf{F} \in \operatorname{Fpc}(f)$ splits into a product of some fixed point classes of $f_{i}$, i.e.,

$$
\mathbf{F}=\mathbf{F}_{1} \times \cdots \times \mathbf{F}_{n}, \quad \operatorname{ind}(f, \mathbf{F})=\operatorname{ind}\left(f_{1}, \mathbf{F}_{1}\right) \cdots \operatorname{ind}\left(f_{n}, \mathbf{F}_{n}\right),
$$

where $\mathbf{F}_{i} \in \operatorname{Fpc}\left(f_{i}\right)$ is a fixed point class of $f_{i}$ for $i=1, \ldots, n$. Moreover,

$$
L(f)=L\left(f_{1}\right) \cdots L\left(f_{n}\right), \quad N(f)=N\left(f_{1}\right) \cdots N\left(f_{n}\right) .
$$

5.2. Proofs of Theorem $\mathbf{1 . 2}$ and Theorem 1.3. Now we can give the proofs of Theorem 1.2 and 1.3. Since the index of fixed points is homotopy invariant, we omit the base points of fundamental groups in the following.

Proof of Theorem 1.2. Let $X=X_{1} \times \cdots \times X_{n}$, and $f: X \rightarrow X$ a homotopy equivalence. Then $f$ induces an automorphism $f_{\pi}: \pi_{1}(X) \rightarrow \pi_{1}(X)$. Note that $\pi_{1}(X)$ is isomorphic to the direct product $\prod_{i=1}^{n} \pi_{1}\left(X_{i}\right)$. By Proposition 2.4, the condition (1) in Theorem 1.2 implies $f_{\pi}=\phi_{1} \times \cdots \times \phi_{n}$ with $\phi_{i}$ an automorphism of $\pi_{1}\left(X_{i}\right), i=1, \ldots, n$. Note that $X_{i}$ is a compact aspherical polyhedron, so $\phi_{i}$ can be induced by a homotopy equivalence $f_{i}: X_{i} \rightarrow X_{i}, i=1, \ldots, n$. Since the product $X$ is also an compact aspherical polyhedron, $f$ is homotopic to the product map $f_{1} \times \cdots \times f_{n}$ which is also a homotopy equivalence. Recall that $X_{i}$ has BIPHE, then the index $\operatorname{ind}\left(f_{i}, \mathbf{F}_{i}\right)$ of any fixed point class $\mathbf{F}_{i}$ of $f_{i}$ has a finite bound $\mathcal{B}_{X_{i}}$ depending only on $X_{i}$. By the product formula of index in Lemma 5.2, we have the index $|\operatorname{ind}(f, \mathbf{F})|<\mathcal{B}_{X}:=\prod_{i=1}^{n} \mathcal{B}_{X_{i}}$ for every fixed point class $\mathbf{F}$ of $f$. Therefore, $X$ has BIPHE.

Proof of Theorem 1.3. Let $M_{1}, \ldots, M_{n}$ be connected closed Riemannian manifolds, each with negative sectional curvature everywhere, and $M=M_{1} \times \cdots \times M_{n}$. Collect together the coordinates corresponding to homotopy equivalent $M_{i}$ 's and present it in the form

$$
M=\prod_{i=1}^{s} M_{i}^{n_{i}} \times \prod_{i=s+1}^{m} M_{i}^{n_{i}}
$$

where $M_{1}, \ldots, M_{s}, 0 \leq s \leq n$, are hyperbolic surfaces, $M_{s+1}, \ldots, M_{m}$ have dimensions $\geq 3, n_{i} \geq 1$ (recall that $n_{i}$ is not the dimension but the number of copies of $\left.M_{i}\right), n_{1}+\cdots+n_{m}=n$ and $M_{i} \not M_{j}$ for $1 \leq i \neq j \leq m$. Then by Example 2.7, the fundamental group $G_{i}:=\pi_{1}\left(M_{i}\right)$ is centerless and indecomposable for $i=1,2, \ldots n$. Therefore,

$$
\pi_{1}(M)=\prod_{i=1}^{s} G_{i}^{n_{i}} \times \prod_{i=s+1}^{m} G_{i}^{n_{i}},
$$


where $G_{i} \not G_{j}$ for $1 \leq i \neq j \leq m$.

For any homotopy equivalence $f: M \rightarrow M, f$ induces an automorphism $f_{\pi}$ of $\pi_{1}(M)$, then by Proposition 2.4. there exist automorphisms $\phi_{i, j} \in \operatorname{Aut}\left(G_{i}\right)$ and permutations $\sigma_{i} \in S_{n_{i}}$, such that

$$
f_{\pi}=\prod_{i=1}^{s}\left(\sigma_{i} \circ \prod_{j=1}^{n_{i}} \phi_{i, j}\right) \times \prod_{i=s+1}^{m}\left(\sigma_{i} \circ \prod_{j=1}^{n_{i}} \phi_{i, j}\right):=\phi \times \psi,
$$

where $\phi=\prod_{i=1}^{s}\left(\sigma_{i} \circ \prod_{j=1}^{n_{i}} \phi_{i, j}\right)$ and $\psi=\prod_{i=s+1}^{m}\left(\sigma_{i} \circ \prod_{j=1}^{n_{i}} \phi_{i, j}\right)$. Recall that $G_{i}$ for $i>s$ is the fundamental group of a Riemannian manifold with dimensions $\geq 3$, then $\psi$ can be induced by a homotopy equivalence $h: \prod_{i=s+1}^{m} M_{i}^{n_{i}} \rightarrow \prod_{i=s+1}^{m} M_{i}^{n_{i}}$. On the other hand, $G_{i}(i \leq s)$ is the fundamental group of a closed hyperbolic surface, so the automorphism $\phi_{i, j} \in \operatorname{Aut}\left(G_{i}\right)$ can be induced by a homeomorphism $f_{i, j}$, and hence $\phi$ is induced by the homeomorphism

$$
g=\prod_{i=1}^{s}\left(\sigma_{i} \circ \prod_{j=1}^{n_{i}} f_{i, j}\right): \prod_{i=1}^{s} M_{i}^{n_{i}} \rightarrow \prod_{i=1}^{s} M_{i}^{n_{i}} .
$$

That is $\phi=g_{\pi}$ and thus $f_{\pi}=g_{\pi} \times h_{\pi}=(g \times h)_{\pi}$. Since $M$ is also aspherical, $f$ is homotopic to the product map $g \times h$. By Theorem 3.6 for every fixed point class $\mathbf{F}$ of $h$, we have $|\operatorname{ind}(h, \mathbf{F})|<\mathcal{B}_{M}$ for some finite bound $\mathcal{B}_{M}$ depending only on $M$.

To complete the proof, by Lemma 5.2 it suffices to show that $\left|\operatorname{ind}\left(g, \mathbf{F}^{\prime}\right)\right|<\mathcal{B}_{M}^{\prime}$ for some finite bound $\mathcal{B}_{M}^{\prime}$ depending only on $M$, for every fixed point class $\mathbf{F}^{\prime}$ of $g$. Since every permutation $\sigma_{i} \in S_{n_{i}}$ is a product of disjoint cycles, and $M_{1}, \ldots, M_{s}$ are hyperbolic surfaces, we can rewritten

$$
g=\prod_{k} g_{k}: \prod_{i=1}^{s} M_{i}^{n_{i}} \rightarrow \prod_{i=1}^{s} M_{i}^{n_{i}}
$$

as a product of finitely many cyclic homeomorphisms $g_{k}$ of products of hyperbolic surfaces. Then by Proposition 4.5, we can choose $\mathcal{B}_{M}^{\prime}=\prod_{i=1}^{s}\left|2 \chi\left(M_{i}\right)-1\right|^{n_{i}}$.

\section{REFERENCES}

[BH] M. Bridson and A. Haefliger, Metric Spaces of Non-Positive Curvature, Grund. Math. Wiss. 319, Springer-Verlag, Berlin-Heidelberg-New York, 1999.

[J1] B. Jiang, Lectures on Nielsen Fixed Point Theory, Contemporary Mathematics vol. 14, American Mathematical Society, Providence (1983).

[J2] B. Jiang, Bounds for fixed points on surfaces, Math. Ann. 311 (1998), 467-479.

[JG] B. Jiang and J. Guo, Fixed points of surface diffeomorphisms, Pac. J. Math. 160 (1) (1993), $67-89$.

[JW] B. Jiang and S. Wang, Lefschetz numbers and Nielsen numbers for homeomorphisms on aspherical manifolds, Topology Hawaii, 1990, World Sci. Publ., River Edge, NJ, 1992, 119-136.

[JWZ] B. Jiang, S.D. Wang and Q. Zhang, Bounds for fixed points and fixed subgroups on surfaces and graphs, Algebr. Geom. Topol. 11 (2011), 2297-2318.

[K1] M. Kelly, A bound for the fixed-point index for surface mappings, Ergodic Theory Dynam. Systems 17 (1997), 1393-1408.

[K2] M. Kelly, Bounds on the fixed point indices for self-maps of certain simplicial complexes, Topology Appl. 108 (2000), 179-196.

[Mc] C. McCord,Estimating Nielsen numbers on infrasolvmanifolds, Pac. J. Math. 154 (1992), 345-368.

[RS] E. Rips and Z. Sela, Structure and rigidity in hyperbolic groups. I, Geom. Funct. Anal. 4 (1994), no. 3, 337-371. 
ON THE BOUNDED INDEX PROPERTY FOR PRODUCTS OF ASPHERICAL POLYHEDRA1

[Z1] Q. Zhang, Bounds for fixed points on Seifert manifolds, Topology Appl. 159 (15) (2012), 3263-3273.

[Z2] Q. Zhang, Bounds for fixed points on hyperbolic 3-manifolds, Topology Appl. 164 (2014), 182-189.

[Z3] Q. Zhang, Bounds for fixed points on hyperbolic manifolds, Topology Appl. 185-186 (2015), 80-87.

[ZVW] Q. Zhang, E. Ventura and J. Wu, Fixed subgroups are compressed in surface groups, Internat. J. Algebra Comput. 25 (5) (2015), 865-887.

[ZZ] Q. Zhang and X. Zhao, Bounds for fixed points on products of hyperbolic surfaces, J. Fixed Point Theory Appl. 21: 6 (2019), 1-11.

School of Mathematics and Statistics, Xi'An Jiaotong University, Xi'An 710049, China

E-mail address: zhangq.math@mail.xjtu.edu.cn

Department of Mathematical Sciences, Xi'an Jiaotong-Liverpool University, Jiangsu, CHINA

E-mail address: shengkui.ye@xjtlu.edu.cn 